\title{
Characterising Aggregate Inter-contact Times in Heterogeneous Opportunistic Networks ${ }^{\star}$
}

\author{
Andrea Passarella and Marco Conti \\ IIT-CNR, Via G. Moruzzi 1, 56124 Pisa, Italy \\ \{a.passarella,m.conti\}@iit.cnr.it
}

\begin{abstract}
A pioneering body of work in the area of mobile opportunistic networks has shown that characterising inter-contact times between pairs of nodes is crucial. In particular, when inter-contact times follow a power-law distribution, the expected delay of a large family of forwarding protocols may be infinite. The most common approach adopted in the literature to study inter-contact times consists in looking at the distribution of the inter-contact times aggregated over all nodes pairs, assuming it correctly represents the distributions of individual pairs. In this paper we challenge this assumption. We present an analytical model that describes the dependence between the individual pairs and the aggregate distributions. By using the model we show that in heterogeneous networks - when not all pairs contact patterns are the same - most of the time the aggregate distribution is not representative of the individual pairs distributions, and that looking at the aggregate can lead to completely wrong conclusions on the key properties of the network. For example, we show that aggregate power-law inter-contact times (suggesting infinite expected delays) can frequently emerge in networks where individual pairs inter-contact times are exponentially distributed (meaning that the expected delay is finite). From a complementary standpoint, our results show that heterogeneity of individual pairs contact patterns plays a crucial role in determining the aggregate inter-contact times statistics, and that focusing on the latter only can be misleading.
\end{abstract}

Keywords: opportunistic networks, analytical modelling.

\section{Introduction}

Foundational results in the area of mobile opportunistic networks have clearly shown that characterising inter-contact times between nodes is crucial Aug07. Kar07. In this paper we thoroughly investigate the dependence between the distributions of individual node pairs inter-contact times and the distribution of the aggregate inter-contact times. Specifically, an individual pair distribution is the distribution of the time elapsed between two consecutive contacts between that pair of nodes. The aggregate distribution is the distribution of inter-contact

\footnotetext{
* This work was partially funded by the European Commission under the FETPERADA SOCIALNETS (FP7-IST-217141), FIRE SCAMPI (FP7-IST-258414), and FET-AWARE RECOGNITION (FP7-IST-257756) Projects.
} 
times of all pairs considered together, i.e. is the distributions of all inter-contact times measured in the network between any two nodes.

A clear understanding of the dependence between the individual pairs and the aggregate distributions is very important, although not achieved in the literature yet. It has been clearly shown that, depending on the distribution of pairs inter-contact times, families of forwarding protocols may produce infinite expected delays Aug07. However, most of the literature has focused on the $a g$ gregate distribution (see Section 2 for a review), assuming it is representative of the individual pairs distributions. This is mainly due to the fact that in real traces, it is much easier to measure and characterise the aggregate distribution than the individual pairs distributions, as gathering enough samples for each and every pair is often very difficult. Aggregate inter-contact times have been frequently found to be distributed according to a power-law with or without exponential cut-off. This has been perceived as a severe challenge for forwarding in opportunistic networks, as an important class of protocols yield infinite expected delay if individual pairs distributions are power-law Aug07.

In this paper we carefully review the hypothesis that the aggregate distribution is representative of individual pairs distributions, by deriving an analytical model that describes the dependence between the two. We consider a general heterogeneous networking environment, in which the individual pairs distributions are all of the same type (e.g., exponential, Pareto,... ), but whose parameters are unknown a-priori. We assume that the rates of the pairs inter-contact times (the reciprocal of the averages) are drawn from a given distribution, which, therefore, determines the specific parameters of each pair inter-contact times. In other words, as the distribution of the rates controls the parameters of the inter-contact times distributions, it allows us to control the type of heterogeneity in the network.

The model described in the paper shows that both the distribution of the rates and the distributions of individual pairs inter-contact times impact on the aggregate distribution. In particular, we use the model to find, among others, the conditions under which the aggregate distribution features the main characteristics often found in traces, i.e. a power-law with or without exponential cut-off. We can summarise the key findings presented in the paper as follows.

- Starting from exponentially distributed individual pairs inter-contact times, the aggregate is distributed exactly according to a Pareto law iff the rates of the pairs inter-contact rates are drawn from a Gamma distribution.

- As an exponential distribution is a special case of a Gamma distribution, Pareto aggregate inter-contact times can result from a network where both the individual inter-contact times and their rates are exponentially distributed.

- When pairs inter-contact times are exponential, and rates are drawn from a Pareto distribution, the asymptotic behaviour of the aggregate distribution (for large inter-contact times) is a power-law with or without exponential cut-off. In particular, the exponential cut-off appears when rates cannot be arbitrarily close to 0 . 
- Under exponentially distributed individual pairs inter-contact times, the distribution of the rates plays a crucial role in generating aggregate inter-contact times featuring a heavy tail. Specifically, whenever rates can be arbitrarily close to 0 , a power-law appears in the aggregate distribution.

Our findings clearly show that relying on the aggregate inter-contact times distribution only for assessing key properties of opportunistic networks is not appropriate in general, and can lead to wrong conclusions. In particular, finding a power-law in the aggregate inter-contact times distribution is not necessarily an indication that individual pairs distributions feature a heavy tail as well, and that therefore forwarding protocols may not converge. On the contrary, the heterogeneity of the network, represented in our study by the distribution of the individual pairs inter-contact rates, plays a crucial role in determining the nature of the aggregate distribution, which can be totally different from the distributions of the individual pairs.

The rest of the paper is organised as follows. We review the relevant state-ofthe-art in Section 2 Then, Section 3 presents the general model describing the dependence between the individual pairs inter-contact times, the distribution of their rates, and the aggregate inter-contact times distribution. In Section 4 we use the model to investigate under which conditions aggregate distributions featuring the main characteristics found in real traces can be generated. We also present simulation results validating our analytical findings. Finally, in Section 5 we draw the main conclusions of this study.

\section{Related Work}

The first work, to the best of our knowledge, that highlighted the importance of inter-contact times for studying opportunistic networks is Aug07. In this work authors show by analysis that a popular family of routing protocols may produce infinite expected delays if individual pairs inter-contact times distributions are heavy tailed. The same work also analyses a set of traces, showing that the aggregate inter-contact times actually follow a power-law distribution. Assuming that the same property holds true for individual pairs as well, authors conclude that those forwarding protocols may not converge in real opportunistic networks.

This very pessimistic result has been somewhat softened by the work in Kar07, where authors re-analyse the same traces and suggest that the aggregate intercontact times distribution might indeed present an exponential cut-off in the tail. Assuming, again, that the same property holds true also for individual pairs, they conclude that forwarding protocols might actually not yield infinite delay. In this work authors discuss the fact that the aggregate and the individual pairs distributions may be different. They propose an initial model for studying the dependence between the two, which we exploit as a starting point in our paper. However, they do not study this aspect further, after checking that, in their traces, a subset of individual pairs inter-contact times are distributed according to a power-law with exponential cut-off. 
These two papers informed most of the subsequent literature, which most of the time assumed that the distributions of individual pairs and the aggregate distribution can be used interchangeably. Only a few papers paid attention to individual pairs distributions. Among them, Con07 analysed another set of popular traces, finding that a significant fraction of pairs inter-contact times may follow exponential, Pareto or log-normal distributions. Authors also provided a model similar in spirit to that presented in our work, in which they analyse conditions under which pairs exponential distributions result in a power-law aggregate. As we highlight in the following, the model in Con07] does not incorporate a fundamental aspect, thus obtaining imprecise results. The work in Gao09. also analyses popular traces, finding that over $85 \%$ of the pairs distributions fit an exponential law, according to a $\chi^{2}$ test. The dependence with the aggregate distribution is not studied, though.

Besides this body of work, most of the literature on opportunistic networks gives for granted that aggregate inter-contact times feature a power-law with exponential cut-off, and do not pay attention to the possible difference of the individual pairs distributions. For example, the vast majority of the mobility models proposed for opportunistic networks share this assumption, and aim at reproducing individual pairs and/or aggregate power-law distributions (e.g., Lee09, Bor09, Bol10, Rhe08). Similarly, other papers try to highlight which characteristics of reference mobility models generate a power-law in individual pairs inter-contact times Cai07, Cai08.

With respect to this body of work, in this paper we provide a thorough analysis of the dependence and the key differences between individual pairs and aggregate inter-contact time distributions, clearly showing that in general the latter cannot be used as a substitute for the former. With respect to the models presented in Kar07 and Con07 we provide much more general and accurate analysis and results. To the best of our knowledge, no previous work has dealt with this specific problem at the level of detail presented here.

\section{Analytical Model of Aggregate Inter-contact Times}

In this section we present an analytical model that describes the dependence between the inter-contact times of individual pairs and the resulting distribution of aggregate inter-contact times. This is the key result that we then exploit in the following analysis.

\subsection{Preliminaries}

As a first step, it is important to recall a result found in Kar07, which shows the relationship between the distribution of individual pairs inter-contact times and the aggregate distribution, in a network where the parameters of the individual pairs distributions are known. Let us assume to monitor individual pairs inter-contact times for a large time interval $T$. Let us denote with $P$ the number of pairs for which at least one inter-contact time is measured over $T$. Moreover, 
denote with $F_{p}(x)$ the CCDF of inter-contact times for pair $p, p \in\{1, \ldots, P\}$, with $n_{p}(T)$ and $N(T)$ the number of inter-contact times of pair $p$ and the total number of inter-contact times over $T$, respectively. Finally, denote with $\theta_{p}$ the rate of inter-contact times for pair $p$ (i.e. the reciprocal of the average intercontact time) and with $\theta=\sum_{p} \theta_{p}$ the total rate of inter-contact times. Then, the CCDF of the aggregate inter-contact times $F(x)$ can be expressed as in the following lemma.

Lemma 1. In a network where $P$ pairs of nodes exist for which inter-contact times can be observed, the CCDF of the aggregate inter-contact times is:

$$
F(x)=\lim _{T \rightarrow \infty} \sum_{p=1}^{P} \frac{n_{p}(T)}{N(T)} F_{p}(x)=\sum_{p=1}^{P} \frac{\theta_{p}}{\theta} F_{p}(x) .
$$

Proof. See [Kar07.

Lemma 1 is rather intuitive. The distribution of aggregate inter-contact times is a mixture of the individual pairs distributions. Each individual pair "weights" in the mixture proportionally to the number of inter-contact times that can be observed in any given interval (or, in other words, proportionally to the rate of inter-contact times).

\subsection{General Results}

In this section we extend the result of Lemma 1 to the case in which the parameters of the individual pairs inter-contact times are not known a priori. Specifically, we consider the general case in which the rates of individual pairs inter-contact times are independent and identically distributed (iid) according to a continous random variable $\Lambda$ with density $f(\lambda), \lambda \geq 0$ (for the generic pair $p, \lambda_{p}$ denotes its rate). We also assume that all individual pairs inter-contact times follow the same type of distribution. For the generic pair $p$, the distribution parameters are set such that the resulting rate is equal to $\lambda_{p}$. Note that we are able to model heterogeneous networks, as inter-contact times distributions of different pairs are in general different, as their rates are different. With respect to the notation used in Section 3.1, we hereafter denote with $F_{\lambda}(x)$ the CCDF of the inter-contact times between a pair of nodes whose rate is equal to $\lambda_{1}^{1}$. Under these assumptions, the CCDF of the aggregate inter-contact times becomes as in Theorem 1 .

Theorem 1. In a network where the rates of individual pairs inter-contact times are distributed with density $f(\lambda)$, the CCDF of the aggregated inter-contact times is as follows:

$$
F(x)=\frac{1}{E[\Lambda]} \int_{0}^{\infty} \lambda f(\lambda) F_{\lambda}(x) d \lambda .
$$

\footnotetext{
${ }^{1}$ Note that, when $F_{\lambda}(x)$ is defined by more than one parameter, additional conditions besides the rate should be identified to derive all parameters. Our analysis holds true for any definition of such additional conditions.
} 
Proof. The complete proof is available in Pas11, while here we provide an intuitive sketch. Formally, we prove the theorem by conditioning $F(x)$ on a particular set of individual pairs inter-contact rates, and applying the law of total probability. Note however that we can also obtain Equation 2 by considering a modified network in which we assume that all rates are possibly available, each with probability $f(\lambda) d \lambda . F(x)$ is thus the aggregate over all such individual intercontact times distributions. As the number of distributions becomes infinite and is indexed by $\Lambda$ (a continuous random variable), the summation in Equation[1 becomes an integral over $\lambda$. Furthermore, the weight of each distribution $\left(\theta_{p}\right.$ in Equation (1) becomes $\lambda \cdot p(\lambda)=\lambda f(\lambda) d \lambda$, while the total rate ( $\theta$ in Equation[1) becomes $\int_{0}^{\infty} \lambda f(\lambda) d \lambda=E[\Lambda]$. The expression in Equation 2 follows immediately.

Note that generalising Lemma 1 as in Theorem 1 results in a much richer tool for understanding the dependence between individual pairs and aggregate intercontact times distributions. Specifically in the model provided by Theorem 1 the individual pairs distributions are not pre-defined, but can be tuned according to the random variable $\Lambda$. This allows us to "steer" and control the heterogeneity of the network. As we show in Section 4, this model allows us to study the relationship between individual pairs and aggregate inter-contact times distributions, by assuming that i) individual pairs are heterogeneous; ii) their inter-contact times follow an arbitrary family of distributions $\left(F_{\lambda}(x)\right)$; and iii) their rates follow another arbitrary distribution $(f(\lambda))$. These degrees of flexibility are not provided by the model in Lemma 1 .

As a final remark, a similar generalisation was also attempted in Con07. However, the formulation in [Con07] is not exact, as it does not take into account the fact that, in the mixture defining $F(x)$, distributions of more frequent contact patterns should "weight more" with respect to distributions of less frequent contact patterns.

\section{Aggregated Inter-contact Times Emerging in Different Heterogeneous Networks}

In this section we exploit the model provided by Theorem 1 to investigate the dependence between the distributions of individual pairs inter-contact times and their aggregate distribution. Specifically, we consider exponentially distributed individual pairs inter-contact times (i.e., we assume that $F_{\lambda}(x)=e^{-\lambda x}$ holds true), and study how the aggregate CCDF $F(x)$ varies for different distributions of the individual pairs inter-contact rates, $f(\lambda)$. Considering exponential individual pairs inter-contact times is sensible, as analysis on traces indicates that this hypothesis cannot be ruled out, in general Gao09, Con07.

\subsection{Preview of the Main Results}

As a preview of the results, we will show that power-law distributions (with or without exponential cut-off) for the aggregate inter-contact times can appear even starting from exponentially distributed individual pairs inter-contact times. 
This is a very interesting outcome, indeed. It clearly indicates that - in general - looking at the aggregate distribution of inter-contact times is not enough for inferring the distributions of individual pairs inter-contact times, and can indeed be misleading. We show, for example, that observing a power-law aggregate distribution with shape $\alpha \in(1,2]$ is not sufficient to conclude that a large family of forwarding protocols yield infinite expected delay Aug07. In such a case, individual pairs inter-contact times may actually be exponentially distributed, which would guarantee finite expected delay. The key reason behind this finding is that when the network is heterogeneous, and not all individual pairs contact patterns are statistically equivalent, the heterogeneity of the individual pairs distributions plays a crucial role in determining the aggregate distribution of the inter-contact times, which may be of a completely different type with respect to the individual pairs distributions.

The detailed results are hereafter presented as grouped in two classes. Firstly, in Section 4.2, we investigate under which conditions the aggregate inter-contact times follow exactly a given distribution. Specifically, we impose that $F(x)$ in Equation 2 is equal to such distribution, and find the corresponding distribution of the individual pairs inter-contact rates $f(\lambda)$. Then, in Section 4.3 we find additional cases in which it is not possible to exactly map a given aggregate distribution $F(x)$ to a specific rate distribution $f(\lambda)$, but it is possible to identify rate distributions such that the tail of the aggregate follows a certain pattern.

As those are among the most interesting cases to study, we focus on aggregate inter-contact times distributed according to i) a power-law, ii) a power-law with exponential cut-off, iii) an exponential law. Proofs are available in Pas11].

\subsection{Exact Aggregate Inter-contact Times Distributions}

First of all, we wish to identify rate distributions $f(\lambda)$ that result in power-law (Pareto) aggregate distributions. From Equation 2, and recalling that we assume individual inter-contact times are exponentially distributed, we have to find $f(\lambda)$ such that

$$
\frac{1}{E[\Lambda]} \int_{0}^{\infty} \lambda f(\lambda) e^{-\lambda x} d \lambda=\left(\frac{b}{b+x}\right)^{\alpha},
$$

where $\alpha$ and $b$ are the shape and scale parameters of the Pareto distribution. Note that in this case we consider the definition of the Pareto distribution in which all positive values are admitted, i.e., $x>0$ holds true. The rate distribution $f(\lambda)$ satisfying Equation 3 is provided by Theorem 22 .

Theorem 2. When individual pairs inter-contact times are exponentially distributed, aggregate inter-contact times are distributed according to a Pareto law with parameters $\alpha>1$ and $b>0$ iff the rates of individual inter-contact times follow a Gamma distribution $\Gamma(\alpha-1, b)$, i.e.

$$
F(x)=\left(\frac{b}{b+x}\right)^{\alpha} \Longleftrightarrow f(\lambda)=\frac{b^{\alpha-1}}{\Gamma(\alpha-1)} \lambda^{\alpha-2} e^{-b \lambda} .
$$

\footnotetext{
${ }^{2}$ A qualitatively similar result was also found in Con07. However, due to the inexact formulation of $F(x)$ highlighted before, the exact result differs.
} 
Theorem 2 is one of the most interesting results of this paper. It has been found in Aug07 that a large family of forwarding protocols yield infinite expected delay when the individual pairs inter-contact time distributions are Pareto with $\alpha \in(1,2]$. Based on this result, it has been common in the literature to assume that, if the aggregate inter-contact time distribution is Pareto with $\alpha \in(1,2]$, those forwarding protocols yield infinite delay. Theorem 2 clearly shows that this is not correct, as aggregate power-laws with $\alpha \in(1,2]$ can be obtained starting from exponential individual pairs inter-contact times. In such a case, the expected delay of forwarding protocols is finite.

As a special case of Theorem 2, the following corollary holds true.

Corollary 1. When individual pairs inter-contact times are exponentially distributed, aggregate inter-contact times are distributed according to a Pareto distribution with parameters $\alpha=2$ and $b>0$ iff the rates of individual inter-contact times follow an exponential distribution with rate $b$, i.e.

$$
F(x)=\left(\frac{b}{b+x}\right)^{2} \Longleftrightarrow f(\lambda)=b e^{-b \lambda} .
$$

Proof. This follows immediately from Equation 4 by recalling that a Gamma distribution $\Gamma(1, b)$ is actually exponential with rate $b$.

Corollary 1 further stresses the result of Theorem 2, stating that a power-law distribution of aggregate inter-contact times can be obtained starting from both exponentially distributed individual pairs inter-contact times and pairs rates.

An interesting physical intuition can be highlighted that justifies the above results. Recall that the inter-contact times aggregate is a mixture of the individual pairs inter-contact times. From a physical standpoint, power-law aggregates means that some inter-contact times in the mixture can take extremely large values, possibly diverging. Intuitively, such a behaviour can therefore be generated irrespective of the distribution of individual pairs inter-contact times, by including in the mixture individual pairs whose inter-contact rate is extremely small, arbitrarily close to 0 . This is exactly the effect of drawing rates from Gamma or exponential distributions, which can admit values of the rates arbitrarily close to 0 . The same physical intuition is also confirmed by other results we present in Section 4.3 .

The final result we present in this section shows under which conditions aggregate inter-contact times follow an exponential distribution, i.e., $F(x)=e^{-\mu x}$. This is shown in Theorem 3 .

Theorem 3. When individual pairs inter-contact times are exponentially distributed, aggregate inter-contact times are distributed according to an exponential distribution with rate $\mu>0$ iff the network is homogeneous, i.e. iff all individual pairs inter-contact times are exponentially distributed with rate $\mu$ :

$$
F(x)=e^{-\mu x} \Longleftrightarrow f(\lambda)=\delta(\lambda-\mu),
$$

where $\delta(\cdot)$ is the Dirac delta function. 
Interestingly, Theorem 3 shows that it is sufficient to look at the aggregate intercontact time distribution only when it turns out being exactly exponential, as, starting from individual pairs exponential distributions, the only possibility is that all pairs inter-contact times are distributed with exactly the same exponential law found in the aggregate.

\subsection{Asymptotic Behaviour of Aggregate Inter-contact Times Distributions}

In this section we present a further set of results characterising the asymptotic behaviour of the aggregate inter-contact times. We still assume that individual pairs inter-contact times are exponential, and study the aggregate when pairs rate are drawn from Pareto distributions. For this set of results we are not able to obtain sufficient and necessary conditions for obtaining a given distribution of the aggregate. However, we are still able to show interesting sufficient conditions for obtaining aggregate distributions that asymptotically decay as a power-law with or without exponential cut-off. These results are quite interesting, as several papers in the literature have observed aggregate distributions whose tail decays as a power-law with exponential cut-off. Note that studying the asymptotic behaviour is relevant, as it is the tail of the inter-contact times distributions that determine the convergence properties of forwarding algorithms Aug07.

Firstly, we assume that individual pairs rates are distributed according to a Pareto distribution whose CCDF is $F(\lambda)=\left(\frac{k}{\lambda}\right)^{\gamma}, \lambda>k$, and derive the asymptotic behaviour of $F(x)$ for large $x$. Note that in this case rates are drawn from a Pareto distribution that does not admit values arbitrarily close to 0 . Theorem 4 provides the expression for $F(x)$.

Theorem 4. When individual pairs inter-contact times are exponentially distributed and rates are drawn from a Pareto distribution $F(\lambda)=\left(\frac{k}{\lambda}\right)^{\gamma}, \lambda>k$, the tail of the aggregate inter-contact times decays as a power-law with exponential cut-off, i.e.:

$$
F(\lambda)=\left(\frac{k}{\lambda}\right)^{\gamma}, \lambda>k \Rightarrow F(x) \sim \frac{e^{-k x}}{k x} \text { for large } x
$$

Two interesting insights can be drawn from Theorem 4. First, an aggregate distribution whose tail decays as a power-law with exponential cut-off can emerge also when individual pairs inter-contact times are exponential. Again, this challenges common hypotheses used in the literature, that assume individual inter-contact times are power-law with exponential cut-off because aggregate inter-contact times are distributed according to this law. Second, this result confirms our intuition about the fact that a key reason for an aggregate distributions with a heavy tail is the existence of individual pairs with inter-contact rates arbitrarily close to 0 . In the case considered by Theorem 4 this is not possible, and indeed the tail of the aggregate inter-contact time decays faster than a power-law. 
Finally, we study the asymptotic behaviour of the aggregate distribution when inter-contact rates are drawn from a Pareto distribution in the form $F(\lambda)=$ $\left(\frac{k}{k+\lambda}\right)^{\gamma}, \lambda>0$. The following theorem holds.

Theorem 5. When individual pairs inter-contact times are exponentially distributed and rates are drawn from a Pareto distribution $F(\lambda)=\left(\frac{k}{k+\lambda}\right)^{\gamma}, \lambda>0$, the tail of the aggregate inter-contact times decays as a power-law with shape equal to 1, i.e.:

$$
F(\lambda)=\left(\frac{k}{k+\lambda}\right)^{\gamma}, \lambda>0 \Rightarrow F(x) \sim \frac{1}{x} \text { for large } x
$$

Theorem 5 confirms once more that the presence of individual pairs with contact rates arbitrarily close to 0 result in heavy tails in aggregate inter-contact times. Again, it also confirms that the presence of even significantly heavy tails (shape equal to 1) in the aggregate inter-contact time distribution is not necessarily an indication that individual pairs distributions also present a power-law.

\subsection{Validation}

In this section we validate the results presented before, by comparing the analytical results with simulations. In our simulation model we consider a network of 150 pairs that meet each other with exponential inter-contact times. Rates are drawn at the beginning of each simulation run according to the specific distribution $f(\lambda)$ we want to test. For each pair we generate at least 100 inter-contact times. Specifically, each simulation run reproduces an observation of the network for a time interval $T$, defined according to the following algorithm. For each pair, we first generate 100 inter-contact times, and then compute the total observation time after 100 inter-contact times, $T_{p}$, as the sum of the pair intercontact times. $T$ is defined as the maximum of $T_{p}, p=1, \ldots, P$. To guarantee that all pairs are observed for the same amount of time, we generate additional inter-contact times for each pair until $T_{p}$ reaches $T$. In this way we generate at

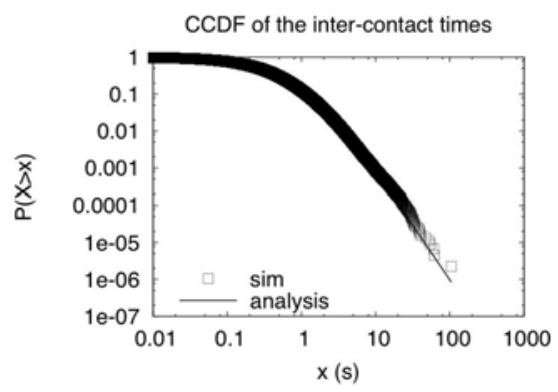

(a)

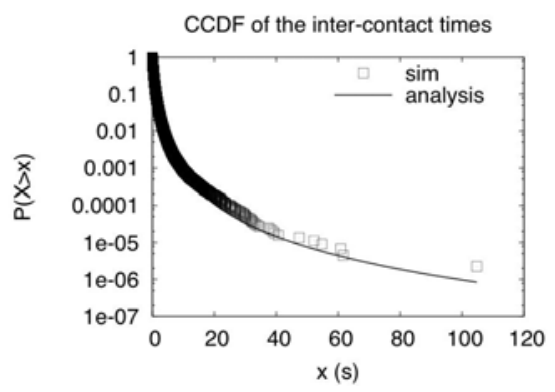

(b)

Fig. 1. $F(x)$, inter-contact rates $\Lambda \sim \Gamma(2,1)(\log \log (\mathrm{a})$ and $\operatorname{lin} \log (\mathrm{b}))$ 


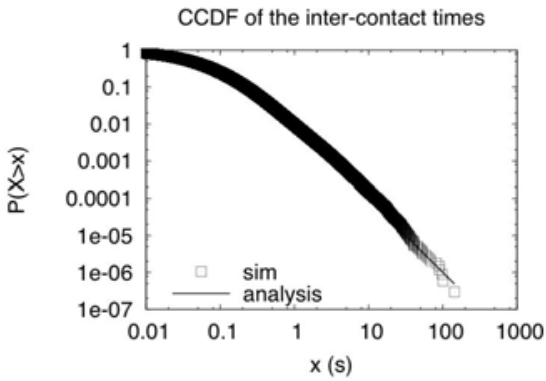

(a)

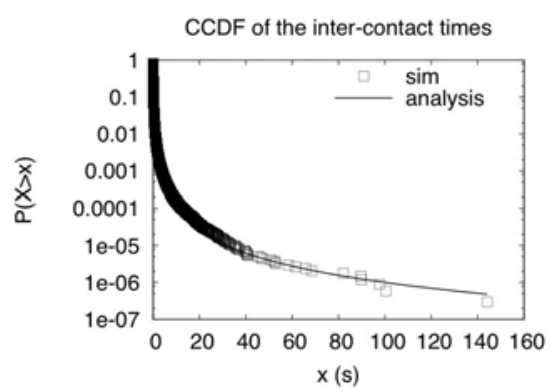

(b)

Fig. 2. $F(x)$, inter-contact rates $\Lambda \sim \operatorname{Exp}(0.1)(\log \log (\mathrm{a})$ and $\operatorname{linlog}(\mathrm{b}))$

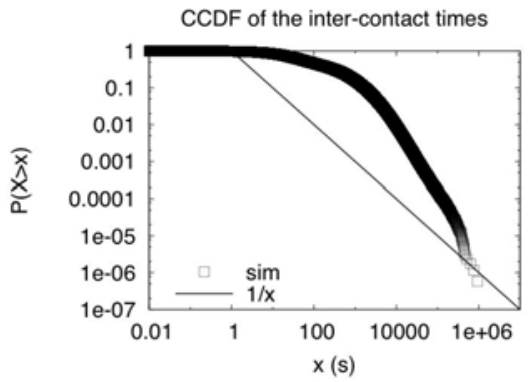

(a)

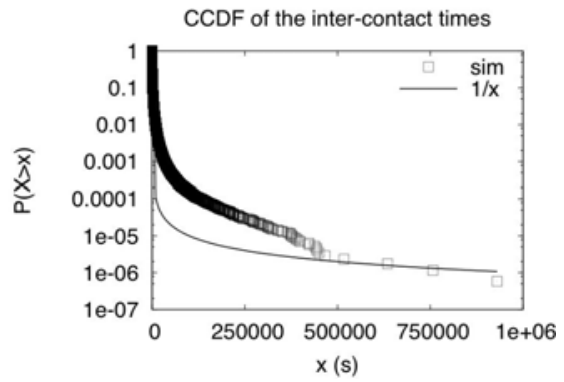

(b)

Fig. 3. $F(x)$, inter-contact rates $\Lambda \sim \operatorname{Pareto}(0.001,2), \lambda>0(\log \log (\mathrm{a})$ and $\operatorname{linlog}(\mathrm{b}))$

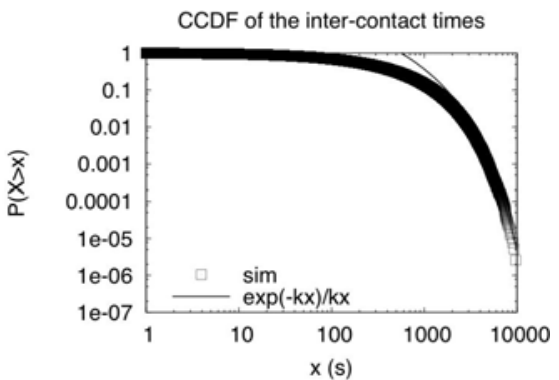

(a)

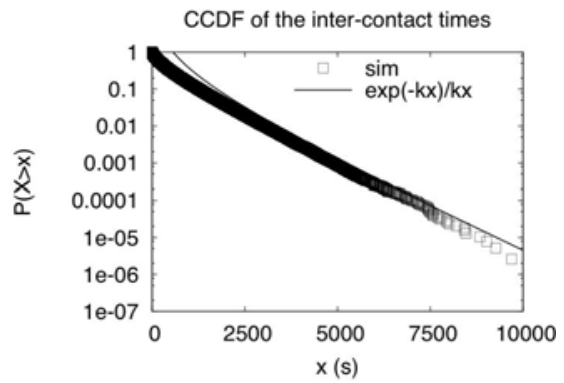

(b)

Fig. 4. $F(x)$, inter-contact rates $\Lambda \sim \operatorname{Pareto}(0.001,2), \lambda>0.001$ (loglog (a) and $\operatorname{linlog}(\mathrm{b}))$

least $150 * 100$ samples of the aggregate inter-contact time distribution, which we consider enough to obtain a reasonably accurate empirical distribution of $F(x)$.

Figure 1 shows the aggregate inter-contact times CCDF $F(x)$ when intercontact rates are drawn from a Gamma distribution $\Gamma(2,1)$ (inter-contact times are reported on the $\mathrm{x}$-axis in seconds). According to Theorem 2 this results in 
aggregate inter-contact times distributed according to a Pareto law with shape $\alpha=3$. The power-law behaviour is clearly highlighted by the less-than-linear decay in the linlog scale (Figure1(b)). It is also clear that simulation and analytical results are in very good agreement.

Figure 2 shows $F(x)$ when the individual pairs inter-contact rates are exponentially distributed with rate $0.1 \mathrm{~s}^{-1}$. Also in this case, according to Corollary 1 , the aggregate inter-contact time follows a Pareto distribution with shape $\alpha=2$. Figure 2 shows that also in this case analytical results are very well aligned with simulations.

Finally, Figure 3 and 4 show $F(x)$ when the pairs rates are distributed according to a Pareto law $F(\lambda)=\left(\frac{k}{k+x}\right)^{\gamma}, \lambda>0$ and $F(\lambda)=\left(\frac{k}{x}\right)^{\gamma}, \lambda>k$, respectively. From Theorems 5 and 4 the key difference is the fact that in the former case rates can be arbitrarily close to 0 , while in the latter case they cannot. The effect on $F(x)$ is to generate a heavy tail decaying as $1 / x$ in the former case, and a light tail decaying as $\frac{e^{-k x}}{k x}$ in the latter. Recall that in these cases the analysis is not able to capture the complete distribution of $F(x)$, but only its asymptotic behaviour for large $x$. Figures 3 and 4 confirm also that in this case analytical and simulation results are aligned.

\section{Conclusions}

In this paper we have investigated through an analytical model the dependence between the distributions of i) individual pairs inter-contact times, ii) the rates of individual pairs inter-contact times, and iii) the aggregate inter-contact times, in mobile opportunistic networks. Understanding this dependence is important, as most of the literature assumes that the aggregate distribution is representative of the individual pairs inter-contact times distributions, and checks network properties that depend on the latter by considering the former.

Our analytical results clearly show that - in general - this approach is not correct. As one of the most popular cases considered in the literature, we have studied under which conditions the aggregate distribution features a heavy tail, with or without an exponential cut-off. We have shown that in heterogeneous networks (i.e., when not all the pairs distributions are the same), heavy tailed aggregate distributions can appear starting from exponentially distributed individual pairs inter-contact times. Therefore, the aggregate distribution is not representative, in general, of the individual pairs distributions, and that focusing on the former to check properties that depend on the latter can thus be misleading.

Furthermore, we have highlighted the key impact of the distribution of the rates of individual pairs inter-contact times on the aggregate distribution. Whenever rates arbitrarily close to 0 are permitted, heavy tails appear in the aggregate, also when individual pairs distributions are light-tailed. This shows the critical role played by the heterogeneity of individual pairs on the aggregate inter-contact times distribution. 


\section{References}

[Abr72] Abramowitz, M., Stegun, I.: Handbook of Mathematical Functions with Formulas, Graphs, and Mathematical Tables. Dover Publications, Mineola (1972) ISBN 978-0-486-61272-0

[Aug07] Chaintreau, A., Hui, P., Crowcroft, J., Diot, C., Gass, R., Scott, J.: Impact of Human Mobility on Opportunistic Forwarding Algorithms. IEEE Trans. on Mob. Comp. 6(6), 606-620 (2007)

[Bol10] Boldrini, C., Passarella, A.: HCMM: Modelling spatial and temporal properties of human mobility driven by users' social relationships. Elsevier Comput. Commun. 33(9), 1056-1074 (2010)

[Bor09] Borrel, V., Legendre, F., Dias De Amorim, M., Fdida, S.: SIMPS: using sociology for personal mobility. IEEE/ACM Trans. Netw. 17(3), 831-842 (2009)

[Cai07] Cai, H., Young Eun, D.: Crossing over the bounded domain: from exponential to power-law inter-meeting time in MANET. In: ACM MobiCom (2007)

[Cai08] Cai, H., Young Eun, D.: Toward stochastic anatomy of inter-meeting time distribution under general mobility models. In: ACM MobiHoc (2008)

[Con07] Conan, V., Leguay, J., Friedman, T.: Characterizing pairwise inter-contact patterns in delay tolerant networks. Autonomics (2007)

[Gao09] Gao, W., Li, Q., Zhao, B., Cao, G.: Multicasting in delay tolerant networks: a social network perspective. In: ACM MobiHoc (2009)

[Kar07] Karagiannis, T., Le Boudec, J.-Y., Vojnovic, M.: Power law and exponential decay of inter contact times between mobile devices. In: ACM MobiCom (2007)

[Lee09] Lee, K., Hong, S., Kim, S.J., Rhee, I., Chong, S.: SLAW: A New Mobility Model for Human Walks. In: INFOCOM (2009)

[Pas11] Passarella, A., Conti, M.: Characterising aggregate inter-contact times in heterogeneous opportunistic networks., IIT-CNR Tech. Rep. 02/201, http://cnd.iit.cnr.it/andrea/docs/net11_tr.pdf

[Rhe08] Rhee, I., Shin, M., Hong, S., Lee, K., Chong, S.: On the Levy-walk Nature of Human Mobility. In: INFOCOM (2008) 